\title{
INTRODUCCIÓN AL DOSSIER TEMÁTICO POLÍTICAS PÚBLICAS Y SOSTENIBILIDAD URBANA EN EL SIGLO XXI
}

Sylvie Nail ${ }^{*}$

En octubre de 2016 se celebró la tercera conferencia de las Naciones Unidas sobre la Vivienda y el Desarrollo Urbano Sostenible (Hábitat III), que tenía como meta definir la hoja de ruta de los próximos veinte años en términos de vivienda y desarrollo urbano. Esta conferencia se realizó menos de un año después de la cop 21 que dio luz al Acuerdo de París firmado por 195 países, un número nunca alcanzado anteriormente en un acuerdo sobre el cambio climático. En esta perspectiva, parecía oportuno que el número 19 de ÓPE$R A$, correspondiente al segundo semestre de 2016, abordara los desafíos, así como algunas propuestas novedosas en sectores claves de las políticas públicas urbanas.

Efectivamente, ya que más de la mitad de la población mundial vive en zonas urbanas y periurbanas, y que la perspectiva es del $70 \%$ para 2050, las ciudades concentran los desafíos (alimentar las poblaciones, asegurar el crecimiento económico y la cohesión social, preservar el medio ambiente, luchar contra el cambio climático, asegurar la equidad y la dignidad, entre otros), así como las oportunidades para inventar modelos alternativos con el propósito de hacer frente a los desafíos y asegurar un acceso a los servicios, todo en un contexto de gran incertidumbre.

Desde que en 1976 se convocó la primera conferencia Habitat, en Vancouver, las ciudades han seguido creciendo, a menudo sin ninguna planeación, especialmente en los países en vía de desarrollo. Por consiguiente, se ha reconocido a nivel internacional el reto que representa la urbanización a una velocidad nunca vista antes, cuyas perspectivas son lúgubres. Debido a la combinación de crecimiento demográfico, desplazamiento por razones de conflictos, de pobreza o de cambio climático, se prevé que las ciudades seguirán atrayendo poblaciones en tal cantidad que el sector formal de la vivienda no las podrá manejar, lo que podría desembocar en la duplicación del

$1 \quad \mathrm{PhD}$ en Estudios ingleses. Profesora de cátedra de Civilización británica en la Universidad de Nantes (Francia); profesora invitada en la Universidad Externado de Colombia. Editora invitada de este número monográfico. https://www.univ-nantes.fr/nail-s/0/fiche__annuaireksup/ [sylvie.nail@uexternado.edu.co]

DOI: http://dx.doi.org/10.18601/16578651.n19.02 
numero de personas que viven en asentamientos informales -de dos a cuatro mil millones, para 2100- (Fry, 2016), lo que constituye un reto impresionante para cualquier modelo de ciudad, aunque sea "inteligente".

La ambición de Habitat III en este contexto era desarrollar "una nueva agenda urbana" y asegurar el compromiso político para "articular la urbanización como una fuerza positiva para las generaciones presentes y futuras, y avanzar en la búsqueda de la igualdad y la prosperidad compartida", al tener en cuenta los desafíos que representan "la ausencia de planificación y de un marco legal adecuado, que llevan a la incesante expansión de las ciudades, al uso intenso de energía y a los alarmantes y peligrosos impactos del cambio climático, a las múltiples formas de inequidad, de exclusión, y a las crecientes dificultades en la oferta de un trabajo digno para todos" (Naciones Unidas, 2014).

Sin la pretensión de agotar un debate de tal complejidad, que se podría abordar por lo menos desde la gobernanza, la tecnología, lo institucional, desde una aproximación sectorial a las políticas públicas y desde las relaciones con el territorio y la naturaleza, los artículos que se publican en este número representan una contribución para profundizar el análisis sobre algunos de los temas pertinentes en el debate actual sobre los componentes de la sostenibilidad urbana para el siglo XXI, más que todo en América Latina. Se abordan específicamente tres temas cruciales: la institucionalidad, la forma urbana y la movilidad.

Gabriel Araya, de la Facultad de Derecho de la Universidad de Chile, abre este número con una contribución sobre el cambio climá- tico y la adopción del derecho internacional sobre esta materia en el Reino Unido, que demuestra concretamente, desde la institucionalidad que, como lo sostiene el autor, "existe una sincronía permanente entre el orden internacional y el ámbito doméstico que se expresa en la creación de una arquitectura institucional y la elaboración de instrumentos, programas y planes, los cuales se insertan en el resto del engranaje jurídico político, prestando particular atención a la política del desarrollo sustentable".

Para reforzar los argumentos de esta primera contribución, el siguiente artículo, a cargo de María de los Ángeles Barrionuevo Mora, de FLACso Ecuador, explica a partir del caso de Quito y de la revisión de los eventos internacionales y alianzas conformadas, cómo la articulación entre los temas ambientales y las políticas públicas depende mucho de la influencia de la política ambiental internacional, así como de la normatividad en cada país. La autora concluye que estas confluyen para permitir (o no) que "ciertas condiciones ambientales sean asumidas y posicionadas como un problema de política pública que debe ser atendido".

$\mathrm{Al}$ aterrizar el marco institucional internacional de los artículos anteriores, la segunda parte del dossier empieza con una contribución de Tomas Neu, arquitecto y magíster en Estudios y Gestión del Desarrollo de la Universidad de los Andes, Colombia, sobre las implicaciones para las políticas públicas de los límites de las urbes, estos "espacios inciertos" (Bertuzzi, 2006) que constituyen el "paisaje intermedio". Gracias a un recorrido histórico y geográfico que muestra cómo los 
territorios urbanos se moldean, estructurándose y desestructurándose, el autor demuestra la importancia de que las políticas públicas se fortalezcan en el tema de sus relaciones con sus alrededores, con el fin de evitar la fragmentación social y espacial y así propiciar un ordenamiento sostenible del territorio. $\mathrm{O}$, como dice Gustavo Wilches Chaux, construir un nuevo tipo de relación entre la ciudad y la región, "y particularmente entre la ciudad y el campo, significa adoptar en la teoría y en la práctica una serie de valores esenciales que deben orientar todas y cada una de las decisiones que se tomen: equidad, solidaridad, reciprocidad, hospitalidad, identidad" (Wilches, 2013).

A continuación, y siguiendo con el tema de la forma urbana, que es una de las claves para fomentar ciudades prósperas y equitativas, el artículo de María Emilia García Schilardi, de la Facultad de Ciencias Económicas de la Universidad Nacional de Cuyo, Argentina, explora los criterios de sustentabilidad urbana a partir del estudio de caso de la ciudad de Mendoza. Ella pone en relieve, de manera cuantitativa, las características de la estructura urbana para concluir que, de acuerdo con los indicadores analizados, "se afirma que el sistema urbano en estudio no presenta ninguna de las características espaciales deseadas de sustentabilidad" y, en consecuencia, no favorece el bienestar de sus habitantes. Esta conclusión se puede entender a la luz del artículo anterior, en la medida en que Mendoza tuvo una "expansión explosiva, discontinua, en forma de mancha de aceite, que invade tierras antes destinadas a la actividad agrícola o que avanza sobre áreas de gran fragilidad ambiental": le faltó precisamente esta mirada crítica holística sobre el territorio, su comportamiento y sus necesidades.

Como recalca un estudio acerca de los componentes de la sostenibilidad, "las formas urbanas no se pueden considerar como 'sostenibles' en el sentido completo de la palabra, si no son aceptadas por la gente como lugares dónde vivir, trabajar e interactuar" (Bramley et al., 2010). Esta segunda parte del dossier se termina con una contribución que hace eco a esa aserción, por Claudia Gabriela Vargas Fernández y Marisol Rodríguez Sosa, del Instituto de Arquitectura, Diseño y Arte de la Universidad Autónoma de Ciudad Juárez, México. Su artículo, titulado "Aceptabilidad social, forma urbana y la sustentabilidad de barrios urbanos en Ciudad Juárez, Chihuahua", pone en relieve, a través de un estudio de caso cuantitativo y cualitativo a nivel de barrio, la relación que existe entre la forma urbana y la aceptabilidad social, entendida como la percepción de unos modos de vida sustentable, el sentido de comunidad y las interacciones sociales. Las autoras enfatizan que para que existan barrios urbanos sustentables se requiere "conocery reconocer las necesidades locales, las deficiencias y carencias que cada habitante, usuario, barrio o grupo de la ciudad requiera, abriendo las posibilidades que pueden brindar las soluciones y oportunidades para cada tipo de interacción entre ellos".

Bajando la lupa de la escala del urbanismo a políticas sectoriales, la tercera y última parte se enfoca sobre uno de los temas más cruciales de la sostenibilidad urbana, que tiene relación tanto con el desarrollo económico como con el bienestar social, sin olvidar la lucha contra 
las emisiones de gases de efecto invernadero: el tema de la movilidad urbana.

El paradigma de la movilidad urbana sostenible requiere, para ser posible, capacidades institucionales y políticas adecuadas, y un marco regulatorio que la contenga. El sexto aporte, de Oscar Alfonso, del Observatorio de la Coyuntura Metropolitana y Municipal (metromun), Facultad de Economía de la Universidad Externado de Colombia, escudrińa las razones por las cuales, a pesar del costo económico, humano y medioambiental del "colapso de la movilidad cotidiana de pasajeros en Bogotá", no se ha podido concretar la decisión de construir un metro en la capital colombiana en 75 años de reflexión sobre el tema. El autor analiza este rezago a la luz de una teoría que explica los rezagos en la inversión productiva, y presenta un balance de los retrasos en algunas experiencias del continente americano. En sus reflexiones finales, el autor sugiere "una línea de acción política para enfrentar el rezago de decisión y, de allí, también los de financiación, para que se logre el metro, agente de la sostenibilidad urbana bogotana”.

Los artículos de este número, y de esta parte sobre la movilidad como elemento clave de la sostenibilidad urbana, culminan con un texto sobre "Regulación de la movilidad urbana en el área metropolitana de Mendoza”, por Lía Martínez, de la Facultad de Ingeniería, ubA, Argentina; María Emilia García Schilardi, de la Facultad de Ciencias Económicas, Universidad Nacional de Cuyo; María Laura Devito, de la Facultad de Arquitectura, Urbanismo y Diseño, Universidad Nacional de Cuyo, y Marisa Díaz, de la Facultad de Cien- cias Políticas y Sociales, Universidad Nacional de Cuyo, Argentina. Su aporte, además de hacer recomendaciones específicas, muestra que también a nivel de una ciudad intermedia, las limitaciones en la regulación, el uso del suelo y la financiación de los transportes públicos explican las falencias y la ausencia de sostenibilidad.

Con estos tres ejes -institucional, territorial y sectorial-se identifican tres dimensiones en las que las políticas públicas, colombianas en particular, se deberían enfocar con más determinación en estos momentos tan cruciales para la paz, en la medida en que "la paz entre humanos tiene como requisito que seamos capaces de pactar la paz con los ecosistemas, con el clima y con el agua. Y esto, a su vez, implica que el desarrollo se convierta en un factor de paz y simbiosis entre lo urbano y lo rural" (Wilches, 2013).

\section{REFERENCIAS}

Bertuzzi, M. L. (2006). Paisajes intermedios. Materiales para la construcción de un paisaje contemporáneo. Santa Fe: Ediciones Universidad Nacional del Litoral.

Bramley, G., Brown, C., Dempsey, N., Power, S. y Watkins, D. (2010). Social acceptability. En Jenks, M. y Jones, C. (eds.). Dimensions of the Sustainable City (pp.105-128). Dordrecht: Springer.

Fry, A. (2016). Cities at risk: Adaptation bridging the formal and informal. En Nail, S. (ed.). Cambio climático. Lecciones de y para ciudades de América Latina. (pp. 199-216). Bogotá: Universidad Externado de Colombia. 
Naciones Unidas (2014). Preparativos para la Conferencia de las Naciones Unidas sobre la Vivienda y el Desarrollo Urbano Sostenible. Informe del Secretario General de la Conferencia (A/CONF.226/ PC.1/4). Nueva York: Naciones Unidas.

Wilches Chaux, G. (2013), ¿Ciudades sostenibles? Semanario Virtual Caja de Herramienta, 360. Viva la Ciudadanía. Recuperado de http://viva.org.co/ cajavirtual/svc0360/articulo09.html 